\title{
HIV/AIDS epidemic in Belarus
}

\author{
Vladimir Eremin ${ }^{1,2^{*}}$, Elena Gasich', Sergei Eremin ${ }^{1}$, Ekaterina Ambarcumian', Vladimir Lukashov ${ }^{3}$ \\ From $16^{\text {th }}$ International Symposium on HIV and Emerging Infectious Diseases \\ Marseille, France. 24-26 March 2010
}

\section{Background}

Main objective of our work is the estimation of an epidemiological situation on HIV/AIDS in Belarus at the present stage.

\section{Methods}

EIA, Western blot, nested RT-PCR, PCR, sequencing, statistical

\section{Results}

The HIV/AIDS epidemic in Belarus can be divided into two stages. The first stage (1986-1996) was characterized by low prevalence and incidence of HIV-1. The total number of HIV-1-infected individuals in this period was 117, of whom 43 were foreigners who have arrived for study or work in Belarus. Since July 1996, the epidemiological situation in Belarus has changed. An HIV-1 outbreak in Svetlogorsk and Zhlobin in 1996-1997 has caused 1021 new HIV-1 infections within a six-month period. This first stage of the epidemic was characterized by:

1. Young men being mainly affected, with $>60 \%$ of new infections being registered among individuals of 1424 years old;

2. Parenteral route of infection (92\% of all transmissions);

3. Epidemiological process being largely limited to the cities of Svetlogorsk and Zhlobin;

4. The outbreak was caused by a subtype A HIV-1 strain.

For October, first, 200910428 cases of a HIV-infection or 107,8 cases per 100 thousand population are officially registered in Belarus. HIV/AIDS Cases are registered more, than in 190 administrative territories.

The current stage of the epidemic is characterized by:
1. The epidemic still affecting young population (individuals of 19-29 years old - around $66 \%$ of new infections);

2. Heterosexual route of transmission becoming dominant (2009 - 77\% of new infections);

3. Geographical expansion - the epidemic is registered in 198 administrative territories with the highest prevalence in Svetlogorsk (3, 100 cases per 100,000 inhabitants);

4. About $95 \%$ of new HIV-1 cases are caused by the "Svetlogorsk" subtype A strain.

With two-three new HIV-1 cases being registered daily in Belarus, mostly among the young generation. Such situation leads to that the quantity of children born to HIV-infected mothers annually increases. Despite of spent preventive therapy, $11 \%$ of such children are HIV-infected.

\section{Discussion}

Thus, at the present stage epidemic VICH/SPID in Belarus is in the concentrated stage, however gradually falls outside the limits risk groups. The risk group includes now young men of sexually active age: 19-29 years. For changing such situation at carrying out of preventive actions it is necessary to give more attention to the given age group. It is necessary to make epidemiological investigations of cases of infection for an establishment of a source of infection, definition to what group of risk it concerns.

\section{Author details}

${ }^{1}$ Research Institute for Epidemiology \& Microbiology, Minsk, Belarus. ${ }^{2}$ D.I. Ivanovsky Institute of Virology, Moscow, Russian Federation. ${ }^{3}$ Academic Medical Center, University of Amsterdam, Amsterdam, The Netherlands.

Published: 11 May 2010

* Correspondence: veremin@mail.ru

${ }^{1}$ Research Institute for Epidemiology \& Microbiology, Minsk, Belarus 